\title{
La evolución del Aprendizaje Integrado de Contenidos y Lenguas Extranjeras (CLIL) en Galicia
}

\section{The CLIL development in Galicia}

\author{
Carmen Calleja Lameiras, Maria del Mar Rodríguez González \\ Centro de Formación e Recursos (Ourense)
}

\begin{abstract}
Resumen
La metodología de Aprendizaje Integrado de Contenidos y Lenguas Extranjeras (CLIL) es una estrategia para incrementar la competencia lingüística en otras lenguas del alumnado, que se ha hecho necesaria para alcanzar las exigencias del presente mundo globalizado en aras de formar ciudadanos plurilingües $y$ con competencias interculturales. Este estudio refleja la evolución en Galicia de las secciones bilingües desde 2007 hasta el presente curso 2014-2015, en continua progresión tanto en la enseñanza pública como en la privada, a pesar de las dificultades propias de cada modalidad que frenan, pero no consiguen detener una realidad que se impone por su propio peso.
\end{abstract}

Palabras clave: CLIL, plurilingüismo, aprendizaje de lenguas, competencia lingüística e intercultural

\begin{abstract}
The CLIL methodology (Content and language integrated learning) is a way to increase proficiency in other languages of our students has become necessary to meet the demands of the present globalized world for multilingual citizens and with intercultural competencies. This study reflects the evolution of bilingual sections in Galicia from 2007 to the present academic year 2014-2015, in continuous progression both public education and private, despite the difficulties of each modality that slow down but fail to stop a reality imposed by its obviousness.
\end{abstract}

Key words: CLIL, language learning, linguistic and intercultural competence, multilingualism

\section{Introducción}

La metodología CLIL (Content and Language Integrated Learning $=$ Aprendizaje integrado de contenidos y lenguas extranjeras) es un modo para incrementar la competencia lingüística en otras lenguas de nuestros alumnos que se ha hecho necesaria para alcanzar las exigencias del presente mundo globalizado para formar ciudadanos plurilingües y con competencias interculturales.

En Galicia su regulación parte desde 2002, con un plan experimental que comienza en 1999 en la educación secundaria, se extiende a los dos primeros cursos de la ESO y ciclos de FP en 2005 y se hace extensible a la primaria en el curso 2006-2007.

Desde entonces, la implantación de este tipo de metodologías ha ido en aumento constante pese a la insuficiente colaboración de la Administración en términos organizativos y de inversión y la resistencia de algunos sectores que veían amenazada la normalización de la lengua gallega en las aulas por la mayor presencia como lenguas vehiculares de las lenguas extranjeras.

Este estudio refleja la evolución en Galicia de las secciones bilingües desde 2007 hasta el presente curso 2014-2015, en continua progresión tanto en la enseñanza pública como en la privada, a pesar de las dificultades propias de cada modalidad que frenan pero no consiguen detener una realidad que se impone por su propio peso.

\section{Método}

En el diseño convergen las bases del conocimiento cualitativo con los aspectos generales de la formación permanente del profesorado. Se trata de una investigación de carácter descriptivo, interpretativo y explicativo, utilizando la revisión documental (datos y cifras).

En consecuencia, se lleva a cabo una investigación evaluativa a través de datos secundarios. La fuente documental utilizada son los registros de datos de la Consellería de Cultura, Educación y Formación Universitaria, recogidos en el Diario Oficial de Galicia y el Portal Educativo, y por tanto, de carácter público.

El procedimiento principal para recabar los datos es la recolección y supervisión de documentos de forma iterativa y recurrente. En primer lugar se procede a registrar la información y vincularla al planteamiento del problema, examinando cómo encaja en la recolección de datos. Así, se reciben datos no estructurados a través de los documentos escritos (observaciones enfocadas, revisiones de bases de datos...) para darles estructura usando como herramienta la "teoría fundamentada" (los hallazgos van emergiendo fundamentados en los datos). A continuación, se realiza un primer nivel de codificación (codificación abierta) para advertir cuestiones relevantes en los datos y analizar esas cuestiones para descubrir similitudes y diferencias, así como estructuras. Finalmente, en estrecha relación con los datos, se obtienen las categorías (conceptos, experiencias, ideas, hechos relevantes y con significado). 


\section{Instrumentos}

Para hacer acopio de los datos, se ha procedido a la realización de una recolección enfocada de los mismos en la propia fuente (http://www.xunta.es/diario-oficialgalicia/) Se hizo una recopilación de datos estadísticos provenientes de fuentes oficiales (datos secundarios) derivados de unidades de análisis individuales (Krysik, 2005). Los investigadores no generaron los datos, pero tienen acceso directo a ellos (registros) y, por lo tanto, los pueden reunir y analizar.

\section{Procedimiento}

El estudio se lleva a cabo a través de una investigación evaluativa, entendida como una estrategia de comprensión, valoración, descubrimiento y construcción de nuevos conocimientos. Así pues, se rastrearon los datos sobre la incorporación de los centros de enseñanza no universitaria a la red de secciones bilingües, durante ocho cursos académicos. En el proceso, para aumentar el rigor científico de la investigación (Hernández y Mendoza, 2008; Hernández, Fernández y Baptista, 2010), se tuvo en cuenta:

- Dependencia: se lleva a cabo un chequeo cruzado del mismo material por parte de varios asesores del Centro de Formación y Recursos, llegando a un $89 \%$ de acuerdo en las unidades, categorías y temas producidos.

- Credibilidad: los datos consultados se contrastan con diversas fuentes donde están registrados (www.edu.xunta.es/centroseducativos/,www.xunta.es/di ario-oficial-galicia, www.edu.xunta-es/fprofe)

- Información y feedback aportada por los centros y su profesorado: con su participación en los planes de formación gestionados desde los Centros de Formación y Recursos, aportan valor sobre la realidad educativa (observación directa).

- Transferencia: contribuye a una nueva visión en la formación del profesorado (aplicable a otras Comunidades Autónomas) y establece pautas para futuros estudios en este campo.

- Confirmabilidad: Rastreo de los datos en su fuente original (www.xunta.es/diario-oficial-galicia) y explicación lógica utilizada para su interpretación.

\section{Resultados}

El incremento de centros con secciones bilingües ha ido en aumento continuo y progresivo a lo largo de los años, aunque ello no es garantía de que la competencia comunicativa en lengua extranjera del alumnado en Galicia progrese en paralelo.

Este despegue se hace más ostensible en los centros privados y concertados, si atendemos al número de secciones solicitadas y autorizadas (un total de 903 secciones desde 2007 hasta la actualidad, lo que nos arroja un porcentaje de incremento de un $291 \%$, frente a las 1746 públicas, que suponen un incremento del 173\%)

Sin embargo, si atendemos al número global de centros que tienen secciones bilingües, parece que el esfuerzo mayor lo soporta la iniciativa pública, con un total de 408 centros públicos implicados frente a 74 de iniciativa privada, de un total de 1005 centros públicos y 310 centros privados, respectivamente.
Tabla 1.

Evolución de las secciones bilingües

\begin{tabular}{ccccccccc}
\hline \multirow{2}{*}{ Curso } & $\begin{array}{l}\text { Secciones } \\
\text { bilingües }\end{array}$ & IP & IS & FP & FS & PS & IS & T \\
\hline \multirow{2}{*}{$\mathbf{0 7 / 0 8}$} & Públicas & 45 & 90 & 1 & 18 & 1 & 0 & $\mathbf{1 5 5}$ \\
& Privadas & 28 & 8 & 0 & 0 & 0 & 0 & $\mathbf{3 6}$ \\
\hline \multirow{2}{*}{$\mathbf{0 8 / 0 9}$} & Públicas & 63 & 133 & 1 & 13 & 1 & 0 & $\mathbf{2 1 1}$ \\
& Privadas & 49 & 9 & 0 & 0 & 0 & 0 & $\mathbf{5 8}$ \\
\hline \multirow{2}{*}{$\mathbf{0 9 / 1 0}$} & Públicas & 52 & 165 & 0 & 13 & 0 & 0 & $\mathbf{2 3 0}$ \\
& Privadas & 50 & 24 & 0 & 0 & 0 & 0 & $\mathbf{7 4}$ \\
\hline \multirow{2}{*}{$\mathbf{1 0 / 1 1}$} & Públicas & 100 & 116 & 0 & 7 & 1 & 0 & $\mathbf{2 2 4}$ \\
& Privadas & 74 & 17 & 0 & 0 & 0 & 0 & $\mathbf{9 1}$ \\
\hline \multirow{2}{*}{$\mathbf{1 1 / 1 2}$} & Públicas & 196 & 66 & 0 & 9 & 2 & 0 & $\mathbf{2 7 3}$ \\
& Privadas & 88 & 21 & 0 & 0 & 0 & 0 & $\mathbf{1 0 9}$ \\
\hline \multirow{2}{*}{$\mathbf{1 2 / 1 3}$} & Públicas & 198 & 75 & 0 & 12 & 2 & 3 & $\mathbf{2 9 0}$ \\
& Privadas & 197 & 37 & 0 & 0 & 0 & 0 & $\mathbf{2 3 4}$ \\
\hline \multirow{2}{*}{$\mathbf{1 3 / 1 4}$} & Públicas & 174 & 85 & 0 & 8 & 1 & 0 & $\mathbf{2 6 8}$ \\
& Privadas & 124 & 47 & 0 & 0 & 0 & 0 & $\mathbf{1 7 1}$ \\
\hline \multirow{2}{*}{$\mathbf{1 4 / 1 5}$} & Públicas & 118 & 71 & 0 & 11 & 5 & 3 & $\mathbf{2 0 8}$ \\
& Privadas & 92 & 38 & 0 & 0 & 0 & 0 & $\mathbf{1 3 0}$ \\
\hline TOTAL & $\mathbf{1 6 4 8}$ & $\mathbf{1 0 0 2}$ & $\mathbf{2}$ & $\mathbf{9 1}$ & $\mathbf{1 3}$ & $\mathbf{6}$ & $\mathbf{2 7 6 2}$ \\
\hline
\end{tabular}

IP: Inglés Primaria; IS: Ingés Secundaria; FP: Francés Primaria; FS: Francés Secundaria; PS: Portugués Secundaria; IS: Italiano Secundaria; T: Total

El marketing hace que no haya un solo centro de iniciativa privada que no quiera gozar del marbete de calidad que parece dar la palabra bilingüe/ plurilingüe en su oferta educativa, de ahí que en este tipo de centros la incorporación ha sido masiva : de un total de 310 centros, se han unido al bilingüismo 74, con 903 secciones bilingües creadas, mientras que en los públicos, de 1005 centros públicos que podrían participar en esta iniciativa, el porcentaje de centros adheridos es de 408 con 1746 secciones, entre primaria y secundaria.

Para ello, han tenido que modificar sus plantillas y las relaciones laborales establecidas en ellas. Más difícil lo han tenido los públicos, donde una de las férreas condiciones que puso la Consellería es que en ningún caso se vería aumentado el número de profesores en la composición de los claustros. Se debe tener presente que en el recuento realizado, del número total de centros de enseñanza no universitaria en Galicia se han descontado las escuelas de educación infantil, las de idiomas, las de Formación Profesional que actualmente no tienen oferta de enseñanza y las de deportes, música y danza.

Tabla 2.

Centros con secciones bilingües

\begin{tabular}{|c|c|c|c|c|c|c|}
\hline Año & $\begin{array}{c}\text { Públ } \\
\text { primar }\end{array}$ & $\begin{array}{c}\text { Públ } \\
\text { secund }\end{array}$ & total & $\begin{array}{c}\text { Priv } \\
\text { primar }\end{array}$ & $\begin{array}{c}\text { Priv } \\
\text { secund }\end{array}$ & total \\
\hline 2007 & 31 & 48 & 79 & 12 & 2 & 14 \\
\hline 2008 & 28 & 35 & 63 & 11 & 2 & 13 \\
\hline 2009 & 17 & 28 & 45 & 13 & 0 & 13 \\
\hline 2010 & 32 & 24 & 56 & 10 & 0 & 10 \\
\hline 2011 & 14 & 5 & 19 & 4 & 1 & 5 \\
\hline 2012 & 52 & 13 & 65 & 15 & 0 & 15 \\
\hline 2013 & 29 & 13 & 42 & 1 & 1 & 2 \\
\hline 2014 & 28 & 11 & 39 & 2 & 0 & 2 \\
\hline total & 231 & 177 & 408 & 68 & 6 & 74 \\
\hline
\end{tabular}

PORCENTAJES con respecto al total de centros

\begin{tabular}{|l|c|}
\hline Total centros públicos & 408 de 1005 \\
\hline Porcentaje públicos & $\mathbf{4 0 , 5 9 \%}$ \\
\hline Total centros privados & 74 de 310 \\
\hline Porcentaje privados & $\mathbf{2 3 , 8 7 \%}$ \\
\hline
\end{tabular}


Además, la Administración pone en marcha una dotación económica anual en función del número de secciones para elaborar y adquirir material didáctico, y una cierta liberación horaria para los coordinadores siempre y cuando sea posible y no lesiva en la organización interna de los centros, con la finalidad de coordinar al profesorado participante y elaborar materiales adaptados a las necesidades que imponen las metodologías CLIL. Asimismo, el profesorado se compromete a incrementar su formación lingüística y metodológica, siempre en horario no lectivo y usando períodos vacacionales y/o de fin de semana.

\section{Discusión y conclusiones}

La oferta formativa de la Consellería consiste en unas xornadas anuales celebradas en fin de semana, cursos de actualización lingüística y comunicativa (CALC) en las escuelas de idiomas para, de un modo acelerado obtener el nivel B2 que exige el MECRL, y un programa de mejora de su competencia lingüística y comunicativa con inmersión en el extranjero (Programa integral de aprendizaje de lenguas extranjeras PIALE)

En los distintos CFR se van organizando cursos de metodología CLIL y talleres de conversación, todo ello con un número limitado de plazas y de carácter voluntario.

En el caso de los centros concertados, no se observa aumento significativo alguno en la participación del profesorado en las actividades formativas, siendo anecdótica su presencia en los cursos de metodología ofertados desde el CAFI y los CFR.

En cuanto a los cursos CALC, un año tras otro se advierte que el número de profesores que obtienen el B2 mediante este sistema es ínfimo.

En el caso del PIALE, las 85 plazas anuales son muy inferiores a las necesidades de la demanda, y la participación un año hace muy difícil poder repetir en ocasiones sucesivas, aun sabiendo que en el tema de las lenguas, uno está siempre en un plano inclinado, de modo que, o avanza continuamente o es imposible mantenerse sin mermar las competencias lingüísticas si se detiene el proceso formativo.

El negocio de las evaluaciones externas (Trinity, Cambridge) prospera gracias al nuevo afán de credencialismo académico que se ha extendido por todo el país, pero una transformación tan profunda no se obtiene con el mero voluntarismo y los barnices que maquillan una realidad ostensible: es imposible acometer una transformación metodológica sin una seria inversión económica, estructural y de tiempo.

El profesorado tiene que recibir una formación mucho más profunda, sistemática e ininterrumpida, y ha de tener los estímulos adecuados para asumir ese compromiso.

Esa formación es bicéfala: por un lado las metodologías para aprender a enfocar la enseñanza y la evaluación usando nuevas pautas: diseño de tareas integradas para la programación por competencias, uso de rúbricas, atención a las inteligencias múltiples, scaffolding learning para que el alumno desarrolle sus estrategias comunicativas con un soporte (andamiaje) temporal que lo conduce a una mayor autonomía en el aprendizaje... Todo esto no se improvisa ni se puede confundir con impartir una materia en otro idioma, que es lo que, en el mejor de los casos, se viene haciendo generalmente.

Por el otro, si el profesorado anda escaso e inseguro en competencia comunicativa en la lengua extranjera, difícilmente podrá ayudar a los alumnos con este objetivo.

Como colofón, no existe un seguimiento del real funcionamiento de dichas secciones, de modo que en cada caso se desarrollan como bien tiene a entender cada cual, desde realizar parte de las tareas en la L2, a limitar su uso al saludo y cuatro o cinco frases rutinarias, a limitar la expresión en las pruebas evaluadoras objetivas de doble alternativa o, como mucho de respuestas múltiples, o directamente, traduciendo los textos e impartiendo en su totalidad la asignatura en otra lengua con los mismos métodos de siempre.

De tal modo que nadie tiene claro si los alumnos salen de ellas defendiéndose mejor en inglés, o sólo conociendo el vocabulario propio de esa materia, sabiendo un poco menos de la asignatura en cuestión dado que gran parte del tiempo la han tenido que emplear en desentrañar un lenguaje que les es ajeno, o si en verdad han desarrollado sus destrezas comunicativas y han aprendido a transferir los inputs de una lengua a otra, a trabajar las distintas inteligencias y ganar en autonomía y estrategias de aprendizaje y expresión...un objetivo muy alto para pretender alcanzarlo con una simple escalerilla de mano...

\section{Referencias}

Council of Europe (2001). Common Eurpean Framework of Referencr for Languages: Learning, teaching, assessment. Cambridge: Cambridge University Press.

Council of Europe (2004). European Language Portfolio. Strasbourg: Cambridge University Press.

Dekeyser, R. y Larson-Hall, J. (2002). What does the critical period really mean? Oxford: OUP.

Deller, S y Price, C.(2007) Teaching other subjects through English. Oxford: Oxford University Press.

Dirección Xeral de Educación, Formación Profesional e Innovación educativa (2011). Plan Galego de Potenciación das Linguas Estranxeiras. Santiago de Compostela. Xunta de Galicia. (DOG, 157, 18/08/2011).

Hernández, R., Fernández, C. y Baptista, P. (2010). Metodología de la investigación. México D.F.: McGRAW-HILL.

Hulstijn, J.H. (1989). Learning words meaning from context: An experimental comparison of the effects of four oriented cues. Artikelen, 34, 13-25.

Mertens, D.M. (2005). Research and evaluation in education and psychology: Integrat ing diversity with quantitative, qualitative, and mixed methods ( $2 \mathrm{a}$ Ed.). Thousand Oaks, CA, EE. UU.: Sage.

Ministerio de Educación y Ciencia (2006). LEY ORGÁNICA 2/2006, de 3 de mayo, de Educación. Madrid. (BOE, 106, 04/05/2006).

Ministerio de Educación y Ciencia (2013). LEY ORGÁNICA 8/2013, de 9 de diciembre, para la mejora de la calidad educativa. Madrid. (BOE, 295, 10/12/2013). 
Ministerio de Educación y Ciencia (2006). Real Decreto 1629/2006, de 29 de diciembre, por el que se fijan los aspectos básicos del currículo de las enseñanzas de idiomas de régimen especial reguladas por la Ley Orgánica 2/2006, de 3 de mayo, de Educación. Madrid. (BOE, 04/01/2007).

Pérez, C. (2005). Content and Language Integrated Learning (CLIL) Teaching materials for the EFL classroom. Barcelona: Universitat Pompeu Fabra.

Pérez, C. (2008) El enfoque integrado de contenidos y lengua en Europa. Aula de innovación educativa, 168, 7-16.

Pérez, C. (2009). To CLIL or not to CLIL. Xornadas CLIL: integrando linguas a través do currículo. Conferencia inaugural. Vigo. Consellería de Educación e Ordenación Universitaria. Xunta de Galicia.

Robinson, P. (1995a). Task Complexity and Second Language Narrative Discourse. Language Learning, 45 (1), 99-140.

San Isidro Agrelo, F.X. (2009): CLIL: Integrando linguas a través do currículo. Santiago de Compostela: Consellería de Educación e Ordenación Universitaria. Xunta de Galicia.

Xunta de Galicia (2011). ORDE do 12 de maio de 2011 pola que se regulan as seccións bilingües en centros sostidos con fondos públicos de ensino non universitario. (DOG, 97, 20/05/2011).

Xunta de Galicia (2011). ORDE do 12 de maio de 2011 pola que se regulan os centros plurilingües na Comunidade Autónoma de Galicia e se establece o procedemento de incorporación de novos centros á Rede de Centros Plurilingües de Galicia. (DOG, 97, 20/05/2011).

Xunta de Galicia (2007) ORDE do18 de abril de 2007 pola que se crea e regula a convocatoria do Plan de seccións bilingües en centros sostidos con fondos públicos de ensino non universitario. (DOG, 87, 07/05/2007).

Xunta de Galicia (2007) RESOLUCIÓN dol3 de xullo de 2007 da Dirección Xeral de Ordenación e Innovación Educativa, pola que se fai pública a relación de centros autorizados para participar no Plan de seccións bilingües en centros sostidos con fondos públicos de ensino non-universitario convocado pola Orde do 18 de abril de 2007. (DOG, 154, 09/08/2007).

Xunta de Galicia (2008) RESOLUCIÓN do 9 de setembro de 2008 da Dirección Xeral de Ordenación e Innovación Educativa, pola que se fai pública a nova relación de centros autorizados no curso 2008-2009 para participar no Plan de seccións bilingües en centros sostidos con fondos públicos de ensino non universitario convocado pola Orde do 18 de abril de 2007. (DOG, 200, 15/10/2008).

Xunta de Galicia (2009) RESOLUCIÓN do 2 de novembro de 2009 pola que se fai pública a nova relación de centros autorizados no curso 2009-2010 para participar no Plan de seccións bilingües en centros sostidos con fondos públicos de ensino nonuniversitario convocado pola Orde do 18 de abril de 2007. (DOG, 221, 11/11/2009).

Xunta de Galicia (2010) RESOLUCIÓN do 11 de novembro de 2010 da Dirección Xeral de Educación, Formación Profesional e Innovación Educativa , pola que se fai pública a nova relación de centros autorizados no curso 2010-2011 para participar no Plan de seccións bilingües en centros sostidos con fondos públicos de ensino non universitario convocado pola Orde do 18 de abril de 2007. (DOG, 226, 24/11/2010).

Xunta de Galicia (2011) RESOLUCIÓN do 9 de setembro de 2011 da Dirección Xeral de Educación, Formación Profesional e Innovación Educativa, pola que se fai pública a autorización da nova relación de seccións bilingües no curso 2011-2012 en centros sostidos con fondos públicos de ensino non universitario reguadas pola Orde do 12 de maio de 2011. (DOG, 204, 25/10/2011).

Xunta de Galicia (2012) RESOLUCIÓN do 10 de setembro de 2012 da Dirección Xeral de Educación, Formación Profesional e Innovación Educativa, pola que se fai pública a autorización da nova relación de seccións bilingües no curso 2012-2013 en centros sostidos con fondos públicos de ensino non universitario reguadas pola Orde do 12 de maio de 2011. (DOG, 191, 05/10/2012).

Xunta de Galicia (2013) RESOLUCIÓN do 16 de setembro de 2013 da Dirección Xeral de Educación, Formación Profesional e Innovación Educativa, pola que se fai pública a autorización da nova relación de seccións bilingües no curso 2013-2014 en centros sostidos con fondos públicos de ensino non universitario reguadas pola Orde do 12 de maio de 2011. (DOG, 193, 09/10/2013).

Xunta de Galicia (2014) RESOLUCIÓN do 19 de setembro de 2014 da Dirección Xeral de Educación, Formación Profesional e Innovación Educativa, pola que se fai pública a autorización da nova relación de seccións bilingües no curso 2014-2015 en centros sostidos con fondos públicos de ensino non universitario reguadas pola Orde do 12 de maio de 2011. (DOG, 185, 29/09/2014). 